\title{
Cocaine-Induced Posterior Reversible Encephalopathy Syndrome (PRES): A Case Report and Review of the Literature
}

\author{
Vijaya Dasari ${ }^{1}$ and Charles D. Donohoe ${ }^{2^{*}}$ \\ ${ }^{1}$ University of Missouri, Kansas City School of Medicine, Kansas City, MO, USA
}

${ }^{2}$ Department of Neurology, Truman Medical Center, Kansas City, MO, USA

\section{Correspondence to: \\ Charles D. Donohoe, MD \\ Truman Medical Center: Department of \\ Neurology, 2301 Holmes St. Suite 519 \\ Kansas City, MO 64108, USA \\ Tel: 816-404-5271, Fax: 816-404-4272 \\ E-mail: charles.donohoe@tmcmed.org}

Received: October 31, 2018

Accepted: December 19, 2018

Published: December 22, 2018

Citation: Dasari V, Donohoe CD. 2018. CocaineInduced Posterior Reversible Encephalopathy Syndrome (PRES): A Case Report and Review of the Literature.J Neuroimaging Psychiatry Neurol 3(2): 30-34.

Copyright: (C) 2018 Dasari and Donohoe. This is an Open Access article distributed under the terms of the Creative Commons Attribution 4.0 International License (CC-BY) (http:// creativecommons.org/licenses/by/4.0/) which permits commercial use, including reproduction, adaptation, and distribution of the article provided the original author and source are credited.

Published by United Scientific Group

\begin{abstract}
Background: Posterior Reversible Encephalopathy Syndrome (PRES) is a clinical-radiological phenomenon that occurs due to a disruption in the autoregulation of the cerebral blood flow resulting in vasogenic edema. It is seen most often in settings of acute hypertension and is often associated with eclampsia. Symptoms that PRES patients commonly present with include seizures, visual disturbances, headache, and altered mental status.
\end{abstract}

Case: We present a patient and review select cases cited in the literature that cocaine, a common drug of abuse, can precipitate hypertension with PRES. Our patient initially presented with severe headache, acute renal failure, hypertension, profound somnolence, and a widespread vasogenic edema after relapsing and using cocaine. Within 24 hours and with control of her blood pressure, her symptoms resolved and she was alert, cognitively intact and free from any identifiable residual effects.

Conclusion: We suggest that this rapid sequence of clinical and neuroimaging abnormalities following cocaine ingestion may not be a rare phenomenon. As has been previously described, the magnitude of PRES related vasogenic edema is not well correlated with either symptom severity or ultimate clinical outcome.

\section{Keywords}

Posterior reversible encephalopathy syndrome, Headache, Somnolence, Hypertension, Vasogenic brain edema

\section{Abbreviation}

PRES: Posterior Reversible Encephalopathy Syndrome

\section{Introduction}

Posterior Reversible Encephalopathy Syndrome (PRES) is a clinicalradiological condition attributed to failure of the autoregulation of blood flow in the brain resulting in disruption of the blood brain barrier, and subsequent subcortical vasogenic edema [1]. The common mediator is the presence of acute hypertension causing hyperperfusion, cerebral blood vessel damage, and resulting edema [2].

This condition is commonly associated with pregnancy related pre-eclampsia and eclampsia, chronic kidney disease, autoimmune disorders, and multiple 
medications but can be seen in a myriad of other medical situations. Our case highlights the clinical and neuroimaging features of PRES induced by cocaine toxicity, a common drug of abuse but one rarely linked to the radiological findings of PRES.

\section{Case Report}

The patient, a 40-year-old female with a past history of inadequately treated hypertension, chronic kidney disease, and cocaine abuse, presented to the emergency department with 'thunderclap' headaches and hypertensive emergency. She had been sober for a period of 3 months but relapsed and admitted to heavy cocaine binging during the days prior to the onset of her symptoms. On admission, her blood pressure was 189/140 and remained elevated with consistent $\mathrm{BP}$ measurements above 200 systolic. She had a right frontal headache for the past 4-5 days that began after she relapsed on cocaine and worsened over the next 36-48 hours. The headache was accompanied by nausea, vomiting, and photophobia without visual loss. She denied any weakness, nuchal rigidity, paresthesias, fever, or chills.

She was also noted to be increasingly somnolent. While she was easily arousable with tactile stimulation, she would repeatedly fall asleep during the exam often in the middle of sentences. She was admitted for hypertensive emergency accompanied by right frontal headache and somnolence.

While the vast majority of PRES patients present with seizures, encephalopathy, depressed consciousness, and disorientation in addition to headache, visual disturbances, and vomiting, this patient was not disoriented and did not have seizures. On physical exam, she had pinpoint pupils, but no focal neurologic deficits were identified, and when aroused, she was oriented to person, place, time, and insightful regarding her behavior of heavy cocaine binging that preceded her admission. Basic laboratory studies including EKG were normal other than elevated serum creatinine 2.58 and low potassium. Chart review showed consistently elevated creatinine levels indicating a history of chronic kidney disease. Her urine drug screen was positive for cocaine and cannabinoids. She had been prescribed amlodipine for her hypertension but had not been taking it for the past 6 months as she lost her insurance and could not afford it.

Neuroimaging including CT of the head demonstrated subtle hypodensities in the left parietal-occipital lobe and right thalamus (Figure 1). MRI brain was recommended. MRI brain without contrast showed extensive increased signal on FLAIR and T2 in cerebellum, pons, midbrain, and thalamus consistent with PRES (Figure 2). There was no restricted diffusion on DWI. Evidence of chronic small vessel ischemic disease was noted as well. Although historically PRES is thought to affect the occipital and parietal region, the increased T2 signal in the cerebellum, brainstem, and basal ganglia as seen in this patient have been shown to be associated with PRES [1]. While no vessel imaging was done in this case, the lack of abnormalities on DWI and $\mathrm{ADC}$ and the fact that the pattern of involvement anatomically does not follow a vascular distribution rules out stroke.

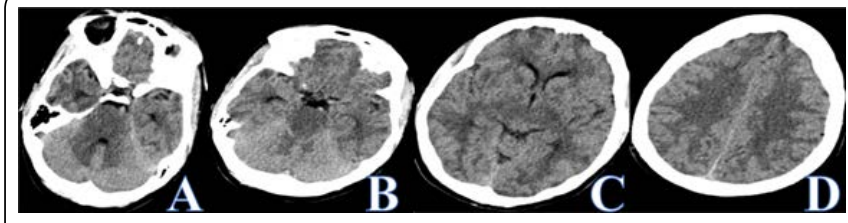

Figure 1: CT performed in the emergency room demonstrates extensive hypodensity in the pons and middle cerebellar peduncle (A), midbrain (B), thalamus and basal ganglia (C), and centrum semiovale (D).

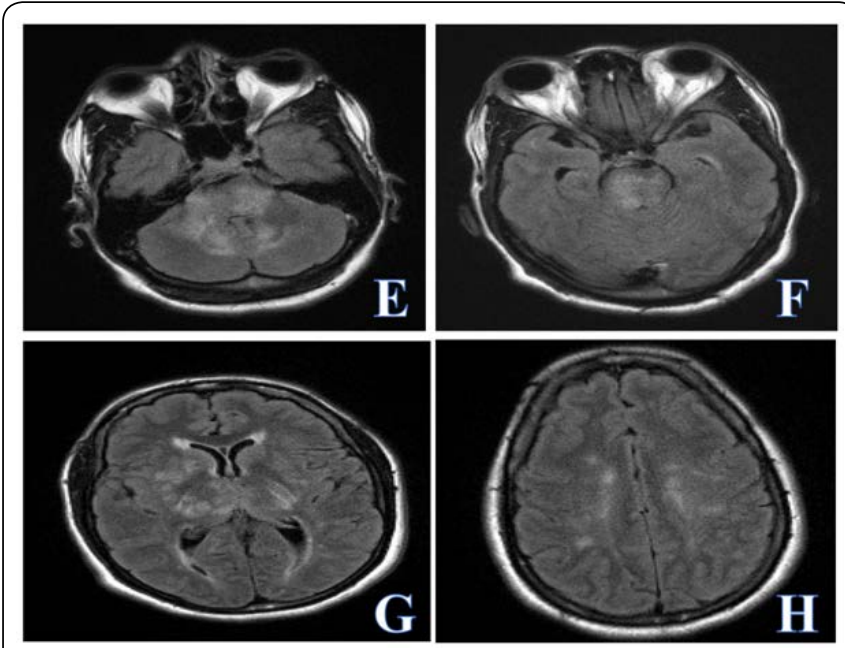

Figure 2: Flair MRI sequences confirm extensive areas of increased signal consistent with vasogenic edema in the pons and middle cerebellar peduncle (E), midbrain (F), thalamus and basal ganglia (G), and the centrum semiovale $\mathbf{( H )}$. There was no associated restricted diffusion or contrast enhancement.

Blood pressure was effectively controlled with IV hydralazine $10 \mathrm{mg}$, amlodipine $10 \mathrm{mg}$ PO, and IV nicardipine $20 \mathrm{mg}$. The IV hydralazine was soon switched to hydralazine $50 \mathrm{mg}$ PO. Two days following admission, her blood pressure had normalized. She was completely alert, cognitively intact, without complaints of headache, and demanding to go home.

\section{Discussion}

\section{Pathophysiology}

Posterior Reversible Encephalopathy Syndrome (PRES) is characterized by dysregulation of cerebral blood flow, which compromises the blood brain barrier and causes vasogenic edema [2]. Although PRES can involve any area of the brain, it preferentially affects the posterior cerebral circulation possibly due an inherent paucity of sympathetic innervation in this territory [3]. While several theories have been proposed as to the cause of cerebral vascular dysautoregulation, there are two theories that have garnered significant support. The first, and most popular, is that in the setting of severe hypertension, the cerebral blood vessels are damaged by resulting hyperperfusion.

This then overwhelms the autoregulatory system of the blood flow in the brain, compromises the blood brain barrier, and leads to subsequent vasogenic edema [1]. While the vast majority of PRES patients present with severe hypertension, PRES patients occasionally present with normotensive blood pressures, leading researchers to search for another cause of 
this cerebral blood vessel dysregulation. One theory to explain this involves immune mediated or inflammatory mediated activation of endothelial cells causing the extravasation of proinflammatory cytokines, leukocyte chemo attractants, and vasoconstrictors. This causes vasoconstriction, hypoperfusion, blood brain barrier dysfunction, and vasogenic edema [3].

The pathophysiology of PRES has not been fully defined and there may be multiple etiologies. While it is often associated with hypertension, there has also been evidence that correlated PRES with autoimmune disorders. One study published in the Mayo clinic proceedings described potential causes of and correlations with PRES. Autoimmune disorders were present in $45 \%$ of the PRES patients, $86 \%$ of patients had acute/severe hypertension, and $57 \%$ of patients were in renal failure at the time of diagnosis [4].

PRES is generally associated with hypertension, sepsis, pre-eclampsia, eclampsia, autoimmune disorders, renal failure, hypercholesterolemia, hypomagnesemia, hypercalcemia, and immunosuppressive/cytotoxic medications [1]. However, it can also be associated with drugs like cocaine and amphetamines, which have sympathomimetic mechanisms that cause vasoconstriction and increased cardiac output leading to severe hypertension [5]. Cocaine blocks the reuptake of monoamines including dopamine, serotonin, and norepinephrine to cause blood vessel vasoconstriction [6]. In long-term cocaine users, the cumulative effect and chronic vasospasm results in a higher likelihood of experiencing vasoconstriction with each use and chronically damages the blood vessel endothelium, promoting atherosclerosis [7].

\section{Clinical presentation}

Symptoms comprising acute encephalopathy associated with PRES include disorientation, headache, vomiting, depressed consciousness, visual disturbance and seizures [8]. The study published in the Mayo clinic proceedings, as previously mentioned, also described how PRES can have a wide range of clinical and radiographic presentations. The most common clinical presentation of PRES involved seizures with seizures in $74 \%$ (primarily generalized tonicclonic), encephalopathy in $28 \%$, headache in $26 \%$, and visual disturbances in $20 \%$ of the patients in the study [4].

The patient presented in this case report came in with headaches and vomiting following cocaine relapse after a period of sobriety. While her past medical history of chronic kidney disease and poorly controlled hypertension predisposed her to developing PRES, the acute hypertensive factors associated with cocaine are more likely the precipitating cause of PRES in this patient. Similarly, not every patient who abuses cocaine will develop PRES, however, those with predisposing factors are more likely to develop this failure of autoregulation.

\section{Imaging}

A diagnosis of PRES is suspected clinically but ultimately rests on neuroimaging. MRI is more sensitive than CT. Although PRES may involve essentially all areas of the brain, the subcortical white matter and cortex of the posterior circulation are preferentially involved, with occasional basal ganglia and brainstem involvement [5]. MRI findings characteristically demonstrate increased signal on T2 and FLAIR with vasogenic edema in the parieto-occipital and posterior temporal lobes, however, imaging abnormalities are not strictly confined to the posterior parieto-occipital and temporal lobes but can be seen diffusely involving the frontal lobes, basal ganglia and cerebellum [1]. One study found that patients with higher BP in presymptomatic period of PRES tended to have a larger vasogenic edematous lesions [9]. Another study published in the Mayo clinic proceedings found that the severity of edema on brain MRI did not correlate clinically with the degree of hypertension or severity of the clinical presentation [4]. Labs studies of blood and CSF are usually not diagnostic. Occasionally signs of endothelial injury including increased LDH, thrombocytopenia, and schistocytes are identified [1].

In the context of status epilepticus, PRES should be suspected if the EEG demonstrates parieto-occipital sharp waves [1].If suspicious clinical or CT findings are present, MRI of the brain should be performed. CT findings are generally subtle and non-diagnostic, while MRI shows conspicuous hyperintense lesions on T2 and FLAIR. The vasogenic edema present in PRES generally does not have restricted diffusion on DWI as is seen in cytotoxic edema associated with acute cerebral infarct [5].

Although PRES is thought to involve only the posterior brain, brain involvement can be diffuse. On Brain MRI in one study, $94 \%$ of patients had parieto-occipital involvement, $77 \%$ had frontal lobe involvement, $64 \%$ had temporal lobe involvement, $53 \%$ had cerebellar involvement, $27 \%$ had brainstem involvement, and 34\% had basal ganglia involvement [4]. Furthermore, on imaging, some unexpected findings included contrast enhancement (21\%), acute hemorrhages (10\%), and restricted diffusion (15\%) [4]. This study pointed out the varied presentations of PRES both radiologically and clinically.

\section{Treatment and outcomes}

The primary treatment of PRES is blood pressure control. Fortunately, the majority of studies describe an excellent prognosis facilitated by prompt diagnosis and progressive treatment of blood pressure leading to complete resolution of both clinical symptoms and abnormalities on brain MRI [5]. However, one study found that PRES patients who had hemorrhage or cytotoxic edema were more likely to experience poorer outcomes [10]. In some rare cases, untreated PRES can cause permanent neurologic deficits due to cerebral ischemia, hemorrhage, and herniation [3].

\section{Other atypical PRES cases}

While cocaine-induced PRES is a rarely documented phenomenon, there are some other atypical PRES cases documented in the literature. One case reported PRES in a patient who had used cocaine and developed severe HTN but did not show classic parieto-occipital edema on MRI but rather had severe vasogenic edema in the brainstem, thalamus, and deep white matter on MRI brain. There were no abnormalities 
on diffusion weighted imaging and these findings normalized on repeat MRI after the hypertension was controlled [11].

Another case described a patient who used mephedrone, a cocaine mimic, and presented with seizures but no focal neurologic deficits. PRES was diagnosed on brain MRI with bi-occipital cortical intensities in the posterior region. These resolved after 10 days on repeat MRI [12]. A case report from Spain also documented PRES in a chronic cocaine user who presented with severe hypertension [13].

Another atypical presentation of PRES was reported in a patient with HIV/AIDS (CD4 count $<10$ ), end-stage renal disease, hypertension and history of intravenous drug use who initially presented with seizure but was oriented and had no focal deficits, so she was discharged. She came back 3 days later with confusion, agitation, and severe HTN without focal deficits or visual disturbances and UDS + for cocaine. CT head was unremarkable, but MRI of the brain showed increased signal on T2 and FLAIR in bilateral cerebral and cerebellar hemispheres and brainstem. With control of BP, repeat MRI on day 7 showed almost complete resolution of the signal abnormalities [3].

\section{Implications}

The diagnosis of PRES can be difficult in patients with multiple comorbidities or mild symptoms. The signs and symptoms can easily be overlooked in an atypical presentation [3]. While there are a few documented cases of PRES in cocaine users, we suggest the majority of cocaine users who develop symptoms such as headache and drowsiness may not come into the hospital for evaluation. This has skewed our experience with CNS toxicity from cocaine toward the age or complications of intraparenchymal hemorrhage, vasospasm, and ischemic stroke. With a normal CT as the primary neuroimaging screening device in the emergency room, its low sensitivity for PRES may hinder its identification in patients abusing cocaine who present with common symptoms including headache and altered mental status. Furthermore, due to atypical presentations in patients with multiple comorbidities, PRES secondary to cocaine neurotoxicity may be more common than previously reported in the literature.

In our patient and review of the literature, cocaine abuse can precipitate acute hypertension and PRES. Our patient initially presented with severe headache, acute on chronic renal failure, hypertension and profound somnolence. Within 24 hours and with control of her blood pressure, her symptoms resolved and she was alert, cognitively intact and free from any identifiable residual effects. We suggest that this rapid sequence of clinical and neuro-imaging abnormalities following cocaine ingestion may not be a unique phenomenon.

We also propose that her prolonged period of sobriety terminating in relapse with a heavy cocaine binge may have been a contributing factor, while also acknowledging that her history of hypertension and chronic kidney disease were predisposing factors to her development of PRES which was ultimately triggered by her cocaine abuse. It is conceivable that transient encephalopathic symptoms in other cocaine abusers evaluated in the emergency room were attributed to cocaine based on a head CT interpreted as normal and a urine drug screen positive for cocaine without the diagnostic refinement provided by MRI in defining the role of PRES. Frequently, a positive UDS can add some degree of bias in medical care and can steer the diagnosis toward attributing the symptoms to drug, while MRI can add important information here.

We are not suggesting that every patient who presents to the ED with a UDS positive for cocaine undergo a brain MRI; we are suggesting that MRI can be a very useful tool when a patient presents with drug use and neurologic symptoms that are difficult to explain. In this case of a diagnostic dilemma, CT is unlikely to identify PRES, and MRI should be done on these patients as cocaine-induced Posterior Reversible Encephalopathy Syndrome may have a higher rate of prevalence than is currently documented.

\section{Conclusion}

Posterior Reversible Encephalopathy Syndrome (PRES) is a clinical-radiologic condition with multiple etiologies felt to be due to disruption of autoregulation of cerebral blood flow and resultant vasogenic brain edema [2]. As described in this case report, cocaine use can result in the development of PRES. We propose that cocaine-induced PRES is more common than is documented in the literature as encephalopathic symptoms in patients who present to the ED with a UDS positive for cocaine are often attributed to the drug use and only a CT head, the standard imaging modality in the ED, is performed. MRI brain is rarely performed in the $\mathrm{ED}$ in patients who present in this manner; however, if it were to be done on patients with these neurologic symptoms after cocaine use, the number of reported cases of cocaine- induced PRES would likely be much higher.

\section{Public health relevance}

The degree of vasogenic edema in Posterior Reversible Encephalopathy Syndrome does not always correlate with clinical condition.

The low sensitivity of CT head for PRES requires the use of MRI brain to properly evaluate the presence and extent of PRES.

The prevalence of cocaine-induced PRES may be higher than is currently reported as cocaine-users with PRES symptoms are less likely to come into the hospital and the standard neuroimaging screening device in the emergency department is CT head, not the more sensitive MRI brain.

\section{Conflict of Interest}

This research was conducted in the absence of any conflicts of interest.

\section{Author Contributions}

VD gathered and analyzed literature concerning PRES 
in relation to the case report. VD drafted and finalized the manuscript and $\mathrm{CD}$ edited and contributed to the text. CD organized the figures and captions involved in the manuscript. All authors reviewed and approved the manuscript.

\section{Acknowledgements}

We would like to thank the patient in the case report as well as the faculty and staff in the Neurology department at Truman Medical Center. We have no sources of funding to report.

\section{References}

1. Lazo KG, Mandel S, Pramanik B, Lee J, DeVita M, et al. 2016. Posterior reversible encephalopathy syndrome (PRES): a case report and review of the literature. Practical Neurology 4: 44-47.

2. Hobson EV, Craven I, Blank SC. 2012. Posterior reversible encephalopathy syndrome: a truly treatable neurologic illness. Perit Dial Int 32(6): 590-594. https://doi.org/10.3747/pdi.2012.00152

3. Bazuaye-Ekwuyasi E, Chow RD, Schmalzle S. 2017. An atypical subacute presentation of posterior reversible encephalopathy syndrome. J Community Hosp Intern Med Perspect 7(4): 269-274. https://doi.org/10 .1080/20009666.2017.1369381

4. Fugate JE, Claassen DO, Cloft HJ, Kallmes DF, Kozak OS, et al. 2010. Posterior reversible encephalopathy syndrome: associated clinical and radiologic findings. Mayo Clin Proc 85(5): 427-432. https://doi. org $/ 10.4065 / \mathrm{mcp} .2009 .0590$
5. Hagan IG, Burney K. 2007. Radiology of recreational drug abuse. Radiographics 27(4): 919-940. https://doi.org/10.1148/rg.274065103

6. Tamrazi B, Almast J. 2012. Your brain on drugs: imaging of drugrelated changes in the central nervous system. Radiographics 32(3): 701719. https://doi.org/10.1148/rg.323115115

7. Stocker S. 1998. Cocaine abuse may lead to strokes and mental deficits. National Institute on Drug Abuse Notes.

8. Sudulagunta SR, Sodalagunta MB, Kumbhat M, Nataraju AS. 2017. Posterior reversible encephalopathy syndrome (PRES). Oxf Med Case Reports 2017(4): 43-46. https://doi.org/10.1093/omcr/omx011

9. Lee MK, Cho YJ, Lee SK, Jung SK, Heo K. 2018. The effect of presymptomatic hypertension in posterior reversible encephalopathy syndrome. Brain Behav 8(8): e01061. https://doi.org/10.1002/ brb3.1061

10. Chen Z, Zhang G, Lerner A, Wang AH, Gao B, et al. 2018. Risk factors for poor outcome in posterior reversible encephalopathy syndrome: systematic review and meta-analysis. Quant Imaging Med Surg 8(4): 421-432. https://doi.org/10.21037/qims.2018.05.07

11. McKinney AM, Short J, Truwit CL, McKinney ZJ, Kozak OS, et al. 2007. Posterior reversible encephalopathy syndrome: incidence of atypical regions of involvement and imaging findings. AJR Am J Roentgenol 189(4): 904-912. https://doi.org/10.2214/AJR.07.2024

12. Omer TA, Doherty C. 2011. Posterior reversible encephalopathy syndrome (PRES) complicating the 'legal high' mephedrone. BMJ Case Rep 2011: bcr0220113904. https://doi.org/10.1136/bcr.02.2011.3904

13. Rodríguez Gómez E, Rodríguez Gómez FJ, Merino MJ, Robledo A, López Domínguez JM, et al. 2001. Reversible posterior leukoencephalopathy, severe hypertension, and cocaine abuse. Nefrologia 21(3): 305-308. 